\title{
CARACTERIZAÇÃO QUALITATIVA DAS MEMBRANAS CATIÔNICAS HDX 100 E PC-SK POR MEV/EDS E FT-IR *
}

\author{
Kayo Santana Barros ${ }^{1}$ \\ Isadora Dias Perez ${ }^{2}$ \\ Jorge Alberto Soares Tenório ${ }^{3}$ \\ Denise Crocce Romano Espinosa ${ }^{4}$
}

Resumo

A busca por processos alternativos para o tratamento de efluentes industriais contendo metais tem aumentado devido às limitações geradas pela precipitação química, como a geração de lama e alto consumo de reagentes. Dentre os métodos avaliados, a eletrodiálise se destaca por possuir ampla aplicabilidade, não gerar mais resíduos e pela possibilidade de recuperação do metal. Entretanto, para garantir sua viabilidade, torna-se necessário estudar a estrutura das membranas empregadas a fim de avaliar 0 comportamento dos íons durante a passagem de um compartimento para outro e definir qual membrana deve ser empregada para cada tipo de separação. Em virtude do exposto, o presente trabalho teve como objetivo caracterizar qualitativamente as membranas trocadoras de cátions HDX 100 e PC-SK por MEV/EDS para avaliar a presença dos elementos químicos em cada uma além de possíveis contaminações, e por FT-IR para determinar suas ligações químicas. De acordo com os resultados obtidos, ambas possuem carbono, oxigênio, enxofre e sódio em suas estruturas confirmando a presença de ácido sulfônico como grupo funcional. As ligações químicas entre os elementos determinados por MEV/EDS presentes nas membranas também foram identificadas por FT-IR. Conclui-se que os métodos empregados no presente trabalho são eficazes para caracterização das membranas avaliadas.

Palavras-chave: Caracterização de Membranas; Polímeros; Troca-iônica.

\section{QUALITATIVE CHARACTERIZATION OF THE CATIONIC MEMBRANES HDX 100 AND PC-SK BY MEV/SEM AND FT-IR}

\begin{abstract}
The interest in alternative processes for the treatment of metal-containing industrial effluents has increased because of the limitations caused by chemical precipitation, as the generation of sludge and reagent consumption. Among the evaluated methods, the electrodialysis has wide applicability, does not generate more residues and enables the metal recovery. However, to guarantee its viability, it is necessary to study the structure of the membranes used in order to evaluate the behavior of ions during the passage from one compartment to another and to define which membrane must be used for each type of separation. The objective of this work was to qualitatively characterize the HDX 100 and PC-SK cation exchange membranes by MEV/SEM to evaluate the presence of the chemical elements in each one, the possibility of contaminations, and by FT-IR to determine chemical bonds. According to the results obtained, both membranes have carbon, oxygen, sulfur and sodium in their structures confirming the presence of sulfonic acid as the functional group. The chemical bonds between the elements determined by MEV/SEM were also identified by FT-IR. We can conclude that the methods used in the present work are effective for the characterization of the evaluated membranes.

Keywords: Membrane Characterization; Polymer; lon-exchange.

1 Engenheiro Químico, Mestre em Engenharia Química, Doutorando do Departamento de Engenharia Química da Universidade de São Paulo, São Paulo - SP, Brasil.

2 Engenheira Ambiental, Mestranda do Departamento de Engenharia Química da Universidade de São Paulo, São Paulo - SP, Brasil.

3 Engenheiro Metalurgista, Doutor, Professor Titular do Departamento de Engenharia Química da Universidade de São Paulo, São Paulo - SP, Brasil.

4 Engenheira Metalurgista, Doutora, Professora do Departamento de Engenharia Química da Universidade de São Paulo, São Paulo - SP, Brasil.
\end{abstract}




\section{INTRODUÇÃO}

O tratamento de efluentes industriais contendo metais tem sido uma das principais preocupações ambientais devido, principalmente, ao aumento do rigor das legislações que determinam a quantidade máxima de suas concentrações para que sejam descartados no meio ambiente. Nos últimos anos, o interesse das indústrias por processos alternativos de tratamento de efluentes tem aumentado devido às limitações apresentadas pelos processos convencionais como a precipitação química, coagulação, complexação, adsorção com carbono ativado, extração por solvente, flotação e cementação [1]. A precipitação química com hidróxido ou sulfato trata-se do principal método utilizado para remoção de metais presentes em efluentes pela sua relativa simplicidade [2], apesar de exigir uma grande quantidade de reagentes capazes de reduzir a concentração dos metais a um nível aceitável para o lançamento do efluente ao meio ambiente e a geração de grande quantidade de lama que exige um tratamento posterior [3].

Dentre os métodos alternativos, o uso de membranas em processos de separação e purificação tem se destacado por não gerar lama, não empregar mais reagentes químicos, aceitar uma ampla faixa de condições operacionais e possuir variadas aplicabilidades como a osmose reversa, nano-, ultra- e microfiltração, pervaporação e eletrodiálise. O sistema de eletrodiálise, por exemplo, é composto por vários compartimentos separados por membranas catiônicas e aniônicas dispostas de forma alternada e possui o campo elétrico como força motriz do transporte iônico [4]. Entretanto, para garantir sua eficiência, é de suma importância avaliar o tipo de membrana que será usada e avaliar sua estrutura para evitar, por exemplo, a queda da permeseletividade pela passagem indesejada de cátions e ânions pelas membranas aniônicas e catiônicas, respectivamente, deposição de matéria orgânica (fouling) [5] e inorgânica (scaling) [6] em sua superfície e um consequente aumento do consumo energético [7].

Em virtude do exposto, o presente trabalho teve como objetivo caracterizar qualitativamente a membrana heterogênea HDX 100 (Hidrodex®) e homogênea PCSK (PCA-Polymerchemie Altmeier GMbH) através de análises de espectroscopia de infravermelhos com transformadas de Fourier (FT-IR) e microscopia eletrônica de varredura equipado com espectroscopia de energia dispersiva (MEV/EDS) para verificar os elementos presentes em cada membrana e suas possíveis ligações químicas. Ambas as membranas são catiônicas e por possuírem baixo custo, alta permeseletividade e alto número de transporte, são frequentemente avaliadas em trabalhos da literatura que envolvem eletrodiálise [4,8-14].

\section{MATERIAIS E MÉTODOS}

A morfologia das membranas HDX 100 e PC-SK foi avaliada por microscopia eletrônica de varredura (MEV) equipado com espectroscopia de energia dispersiva (EDS). Para as leituras, foi utilizada uma tensão de aceleração de $15 \mathrm{keV}$ e distância de trabalho de $13 \mathrm{~mm}$. As possíveis ligações químicas entre os elementos identificados por MEV/EDS foram analisadas por espectroscopia de infravermelhos com transformadas de Fourier (FT-IR). Os espectros foram coletados por uma média de 64 scans com resolução de $4 \mathrm{~cm}^{-1}$ e gravados entre $600-4000 \mathrm{~cm}^{-1}$.

Antes das análises, as membranas foram lavadas e deixadas em água destilada por $24 \mathrm{~h}$. Em seguida, as amostras foram deixadas por mais $24 \mathrm{~h}$ em estufa a $60^{\circ} \mathrm{C}$. 


\section{RESULTADOS E DISCUSSÃO}

\subsection{MEV/EDS}

As Figuras 1 a e b apresentam as imagens de elétrons retroespalhados obtidas por microscopia eletrônica de varredura das membranas HDX 100 e PC-SK, respectivamente, enquanto as Figuras $2 \mathrm{a}$ e $\mathrm{b}$ apresentam os espectros obtidos por EDS para as duas membranas avaliadas.

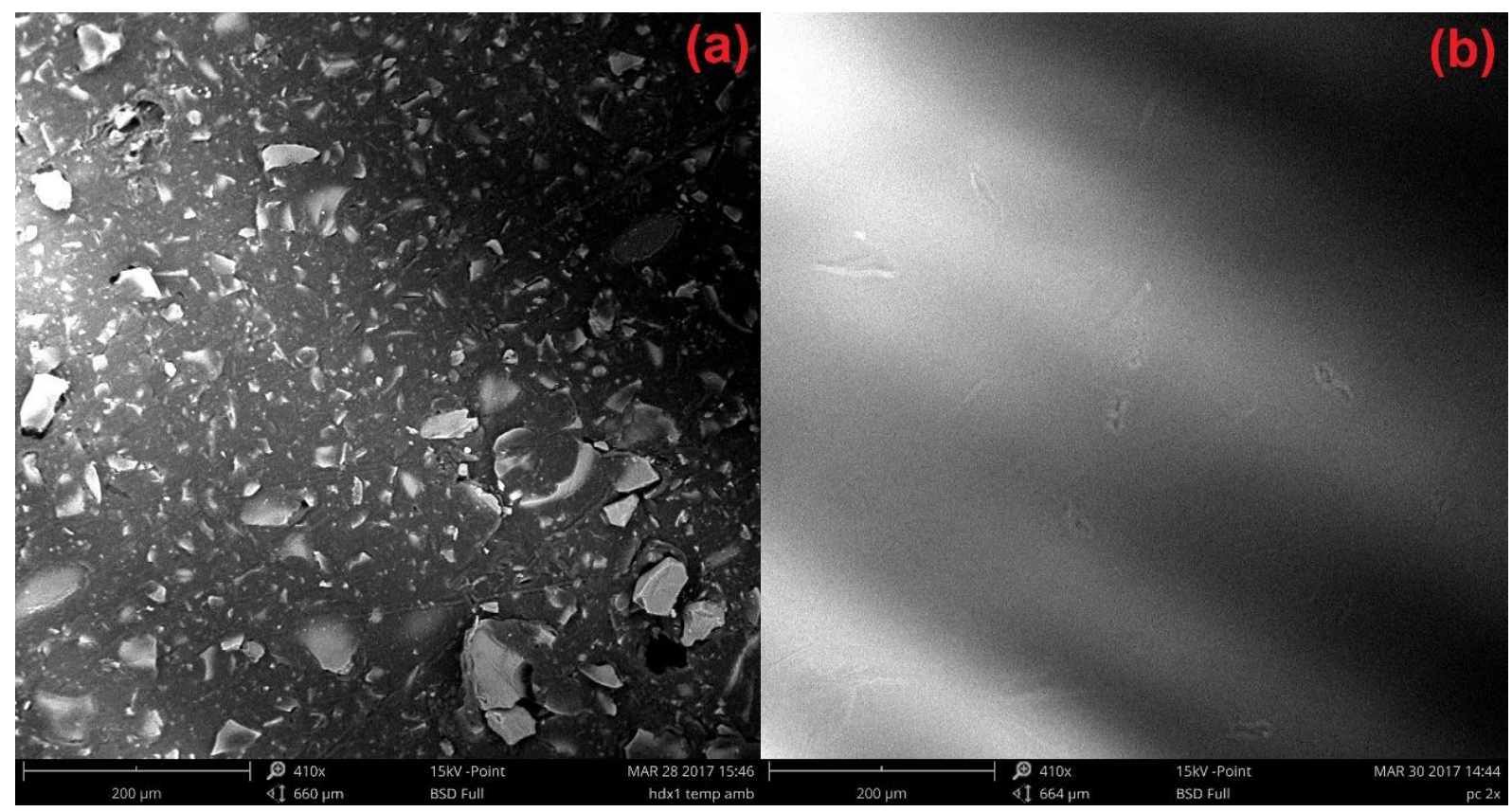

Figura 1 - Imagens de elétrons retroespalhados obtidos em MEV da membrana a) HDX 100 e b) PC-SK.
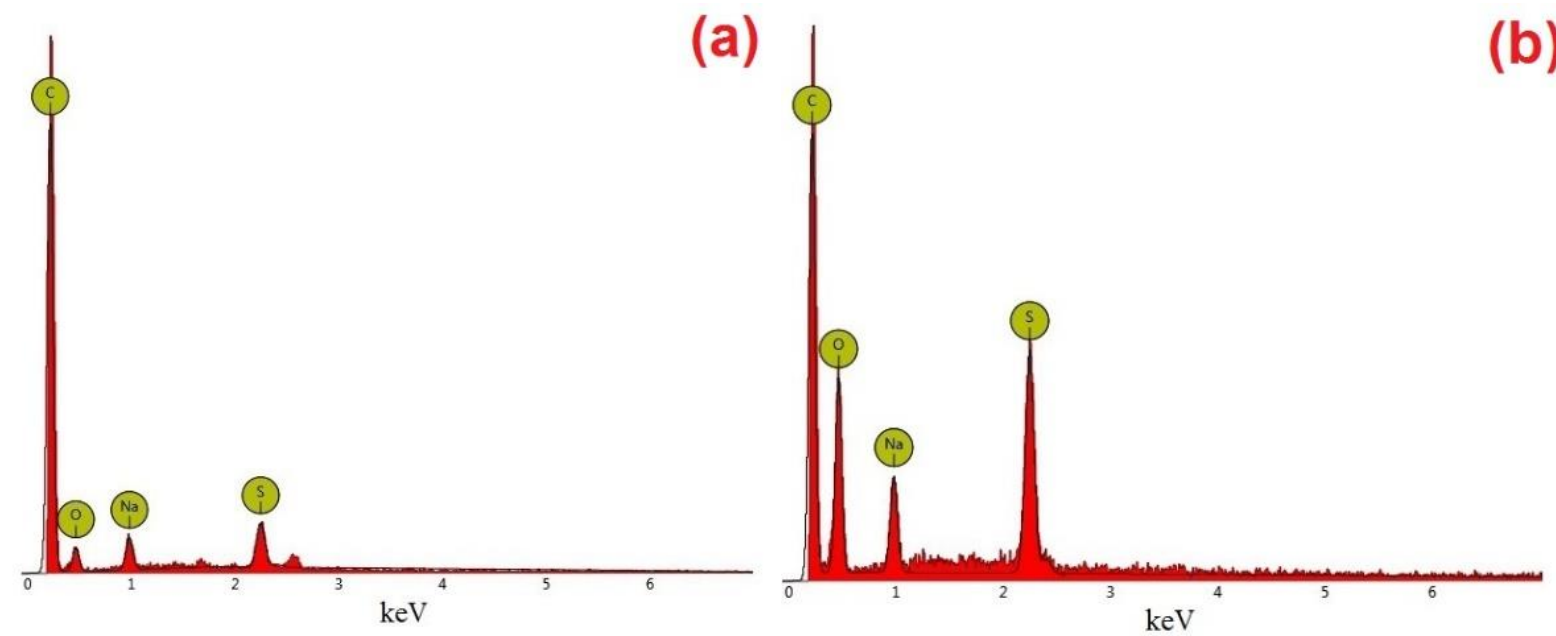

Figura 2 - Espectros de EDS da membrana a) HDX 100 e b) PC-SK. 
A Figura 1a confirma a heterogeneidade da membrana HDX pela presença de aglomerados dispersos de partículas trocadoras de íons, enquanto a Figura $1 \mathrm{~b}$ confirma a homogeneidade da PC-SK, sugerindo assim que a fração de área condutora da PC-SK seja superior à da HDX. Os espectros apresentados nas Figuras 2 a e b mostram que ambas as membranas possuem os mesmos elementos químicos em suas estruturas: carbono, oxigênio e enxofre provenientes do polímero e do ácido sulfônico, o qual confere às membranas a propriedade de trocar cátions no processo de eletrodiálise. A presença do sódio também foi observada na análise já que este elemento se encontra ligado ao $-\mathrm{SO}_{3}$ como co-íon do grupo funcional.

\subsection{FT-IR}

A Figura 3 apresenta os espectros de infravermelhos com transformadas de Fourier (FT-IR) da membrana HDX 100.

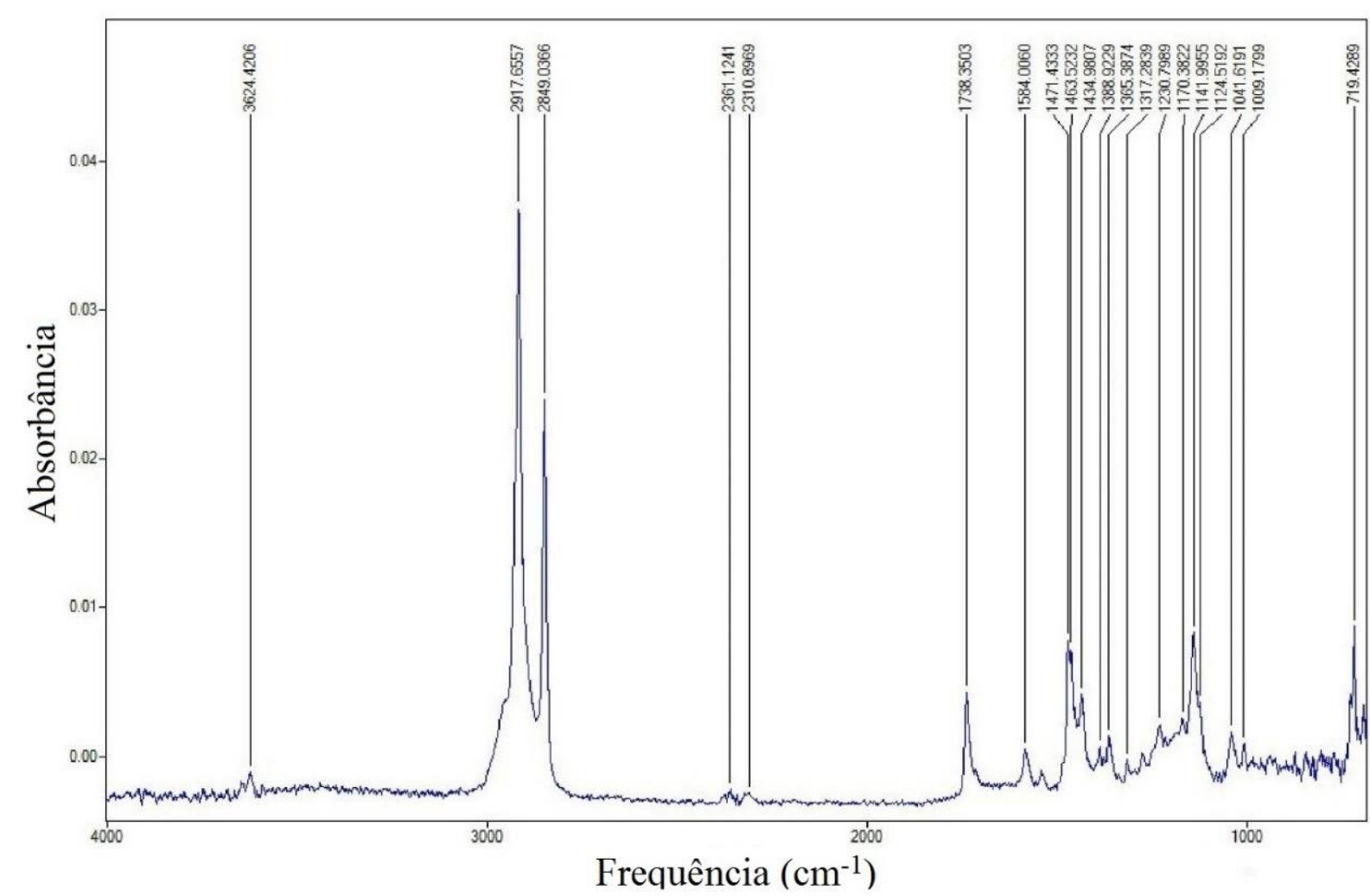

Figura 3 - Espectro de infravermelho obtido por FT-IR da membrana HDX 100.

O pico referente à frequência $3624 \mathrm{~cm}^{-1}$ está relacionado à vibração de estiramento (stretching) do grupo $\mathrm{OH}^{-}$[15], enquanto que em 2918 e $2849 \mathrm{~cm}^{-1}$ os picos estão associados a vas,s $\left(\mathrm{CH}_{2}\right)$, respectivamente [16]. Já os picos referentes a 2361 e $2310 \mathrm{~cm}^{-1}$ se devem ao $\mathrm{CO}_{2}$ presente no ar [17]. Os picos em 1738 e $1584 \mathrm{~cm}^{-}$ 1 estão relacionados ao estiramento dos grupos $\mathrm{C}=\mathrm{O}$ e $\mathrm{C}=\mathrm{C}$ da cadeia polimérica e dos anéis aromáticos, respectivamente [18]. Em $1471 \mathrm{~cm}^{-1}$ o pico se deve ao $\delta\left(\mathrm{CH}_{2}\right)$ [16]. Já em 1464 e $1435 \mathrm{~cm}^{-1}$ os picos estão relacionados à deformação (bending) do $\mathrm{CH}_{2}[19,20]$, enquanto os picos em 1389 e $1365 \mathrm{~cm}^{-1}$ são característicos de $\delta_{\mathrm{s}}\left(\mathrm{CH}_{3}\right)$ [15] e $\delta \mathrm{C}-\mathrm{H}$ [17], respectivamente. Os picos em 1317 e $1231 \mathrm{~cm}^{-1}$ se devem ao vSO 2 
do ácido sulfônico [15]. Em $1170 \mathrm{~cm}^{-1}$ o pico está associado ao $-\mathrm{SO}_{3}{ }^{-}$[20,21] enquanto o pico a $1142 \mathrm{~cm}^{-1}$ é devido a $\mathrm{v}_{\mathrm{s}}\left(\mathrm{SO}_{2}\right)$ [15]. Em $1125 \mathrm{~cm}^{-1}$ o pico ocorre pela vibração de estiramento do S-O [20,22]. O pico em $1042 \mathrm{~cm}^{-1}$ está relacionado ao grupo $\mathrm{S}=\mathrm{O}$ [18,20]. Por fim, os picos em 1009 e $719 \mathrm{~cm}^{-1}$ estão relacionados ao grupo $\mathrm{SO}_{3}^{-}$[20] e à vibração do tipo rocking do $\mathrm{CH}_{2}[15,19]$, respectivamente.

A Figura 4 apresenta os espectros obtidos por FTIR da membrana PC-SK.

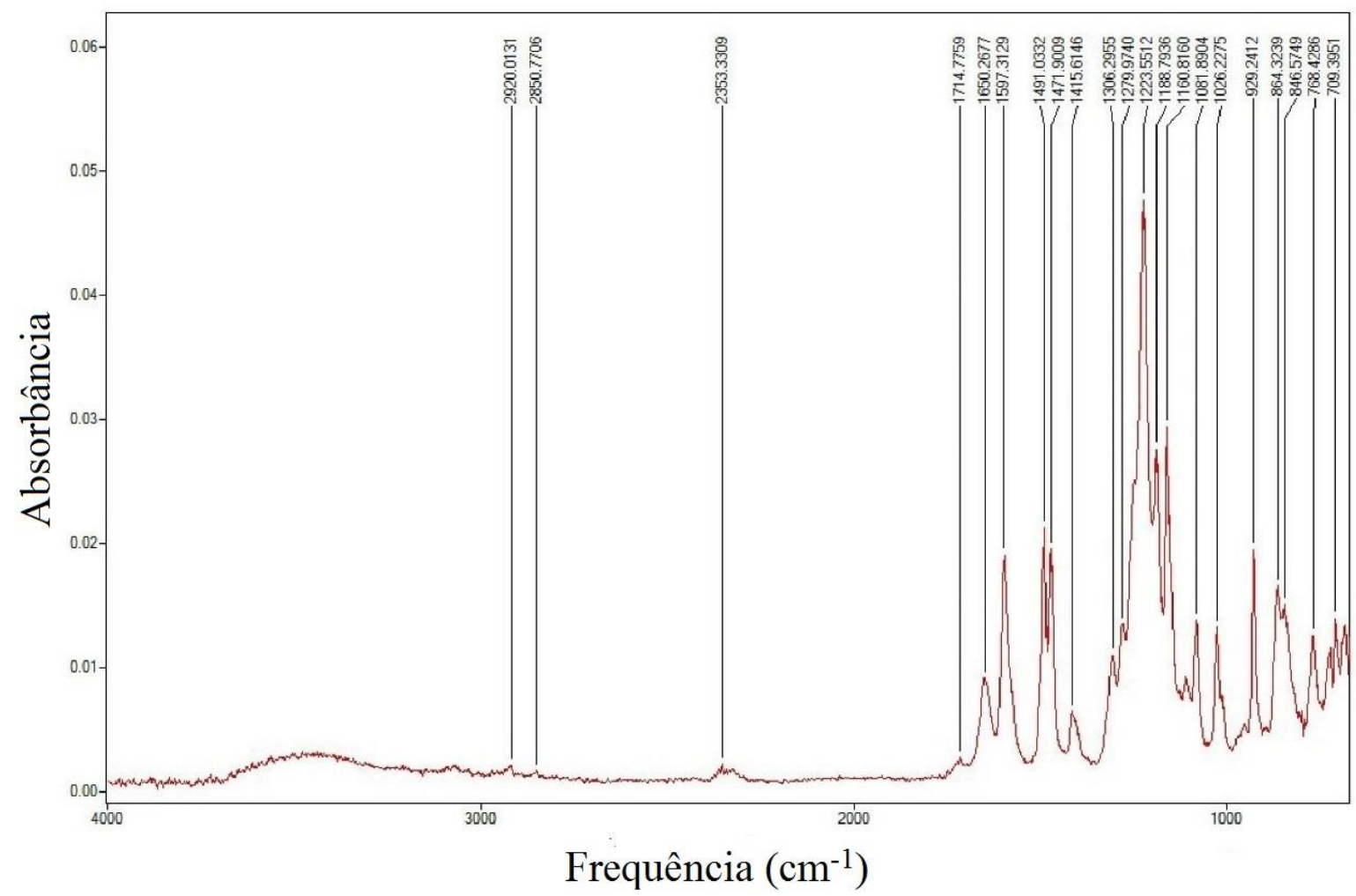

Figura 4 - Espectro de infravermelho obtido por FT-IR da membrana PC-SK.

Em 2920, 2850 e $2353 \mathrm{~cm}^{-1}$ os picos apresentados se devem ao estiramento assimétrico do $\mathrm{CH}_{2}$ [20], $\mathrm{v}_{\mathrm{s}} \mathrm{CH}_{2}$ [15] e à presença do $\mathrm{CO}_{2}$ do ar [15,17], respectivamente. $\mathrm{O}$ pico em $1715 \mathrm{~cm}^{-1}$ está relacionado ao grupo $\mathrm{C}=\mathrm{O}$ da estrutura da matriz polimérica [23]. Em 1650, 1597 e $1491 \mathrm{~cm}^{-1}$ os picos estão associados à vibração dos anéis aromáticos [18,20]. Os picos da banda 1472 e $1416 \mathrm{~cm}^{-1}$ são referentes à vibração do $\mathrm{CH}_{2}$ do tipo scissor [15] e à vibração dos anéis aromáticos [22], respectivamente. Em $1306 \mathrm{~cm}^{-1}$ o pico se deve à vibração de estiramento do C$\mathrm{H}$ [24] e em $1280 \mathrm{~cm}^{-1}$ ao estiramento do C-O [25]. O pico em $1224 \mathrm{~cm}^{-1}$ pode ser devido ao $\mathrm{v}\left(\mathrm{SO}_{2}\right)$ [15], à deformação do $\mathrm{C}-\mathrm{H}$ no plano [26] e à deformação do $\mathrm{C}-\mathrm{C}$ no plano [22]. O pico em $1189 \mathrm{~cm}^{-1}$ está relacionado à vibração de estiramento assimétrica do ácido sulfônico [21], enquanto que o de $1161 \mathrm{~cm}^{-1}$ está associado à deformação do C-H dos anéis [18]. Os picos em 1082 e $1026 \mathrm{~cm}^{-1}$ podem estar relacionados ao grupo $S=O$ provenientes do ácido sulfônico $[15,18]$. Em $929 \mathrm{~cm}^{-1} 0$ pico é devido ao grupo $\mathrm{OH}^{-}$[15], enquanto os picos em 864, 847 e $768 \mathrm{~cm}^{-1}$ se devem a v(S-O) [15]. Finalmente, o pico em $709 \mathrm{~cm}^{-1}$ pode ocorrer pelo $\mathrm{v}(\mathrm{S}-\mathrm{O})$ [15] ou pelo grupo $\mathrm{C}-\mathrm{H}[26]$. 


\section{CONCLUSÃO}

A caracterização das membranas catiônicas comerciais HDX 100 e PC-SK foram realizadas através de MEV/EDS e FT-IR. De acordo com os resultados obtidos, a HDX 100 é de fato heterogênea devido à presença de aglomerados dispersos de partículas trocadoras de íons mostrados nas imagens de MEV, enquanto a PC-SK é homogênea, sugerindo assim que a fração de área condutora da PC-SK seja superior à da HDX. Já nos espectros do EDS foi possível verificar a presença de carbono, oxigênio e enxofre provenientes do ácido sulfônico e da matriz polimérica, além do sódio por este elemento se tratar do co-íon ligado ao grupo trocador de cátions enquanto não ocorre passagem de corrente elétrica. Através da análise de FT-IR foi possível verificar as ligações químicas entre os elementos identificados com $\mathrm{MEV/EDS}$, assim como os picos referentes a cada tipo de vibrações de deformação e estiramento dos grupos presentes nas membranas envolvendo carbono, hidrogênio, oxigênio e enxofre.

Por fim, conclui-se que os métodos empregados no presente trabalho para a caracterização qualitativa de membranas são eficazes e bastante úteis em estudos de avaliação de transporte iônico. Para os trabalhos futuros, sugere-se que seja feita uma análise quantitativa dos elementos químicos, além da distribuição dos grupos funcionais.

\section{Agradecimentos}

Os autores agradecem ao Conselho Nacional de Desenvolvimento Científico e Tecnológico (CNPq) e à Fundação de Amparo à Pesquisa do Estado de São Paulo (Fapesp processo no 2012/51871-9).

\section{REFERÊNCIAS}

1 Ogutveren UB, Koparal S, Ozel E. Electrodialysis for the removal of copper ions from wastewater, J. Environ. Sci. Heal. Part A-Environ. Sci. Eng. 2008:32:749761.

2 Fu F, Wang Q. Removal of heavy metal ions from wastewaters: A review, J. Environ. Manage. 2011;92:407-418.

3 Barakat MA. New trends in removing heavy metals from industrial wastewater, Arab. J. Chem. 2011;4:361-377.

4 Korzenowski C, Rodrigues MAS, Bresciani L, Bernardes AM, Ferreira JZ, Purification of spent chromium bath by membrane electrolysis, J. Hazard. Mater. 2008;154:960-967.

5 Zuo W, Zhang G, Meng Q, Zhang H. Characteristics and application of multiple membrane process in plating wastewater reutilization, Desalination. 2008;222:187-196.

6 Nikonenko VV, Kovalenko AV, Urtenov MK, Pismenskaya, ND, Han J, Sistat P, Pourcelly G, Desalination at overlimiting currents: State-of-the-art and perspectives, Desalination. 342;2014:85-106.

7 Marder L, Ortega Navarro EM, Perez-Herranz V, Bernardes AM, Ferreira JZ. Evaluation of transition metals transport properties through a cation exchange membrane by chronopotentiometry, J. Memb. Sci. 284;2006:267-275. 
8 Martí-Calatayud MC, Buzzi DC, García-Gabaldón M, Bernardes AM, Tenório JAS, Pérez-Herranz $\mathrm{V}$. Ion transport through homogeneous and heterogeneous ionexchange membranes in single salt and multicomponent electrolyte solutions, J. Memb. Sci. 2014;466:45-57.

9 Martí-Calatayud MC, Buzzi DC, García-Gabaldón M, Ortega E, Bernardes AM, Tenório JAS, Pérez-Herranz V. Sulfuric acid recovery from acid mine drainage by means of electrodialysis, Desalination. 2014;343:120-127.

10 Marder L, Bittencourt SD, Zoppas Ferreira J, Bernardes AM. Treatment of molybdate solutions by electrodialysis: The effect of $\mathrm{pH}$ and current density on ions transport behavior, Sep. Purif. Technol. 2016;167:32-36.

11 Zhang Y, Van der Bruggen B, Pinoy L, Meesschaert B. Separation of nutrient ions and organic compounds from salts in $\mathrm{RO}$ concentrates by standard and monovalent selective ion-exchange membranes used in electrodialysis, J. Memb. Sci. 2009;332:104-112.

12 Zhang YF, Liu L, Du J, Fu R, Van der Bruggen B, Zhang Y. Fracsis: Ion fractionation and metathesis by a NF-ED integrated system to improve water recovery, J. Memb. Sci. 2017;523:385-393.

13 Cassady HJ, Cimino EC, Kumar M, Hickner MA. Specific ion effects on the permselectivity of sulfonated poly(ether sulfone) cation exchange membranes, J. Memb. Sci. 2016;508:146-152.

14 Rottiers T, Ghyselbrecht K, Meesschaert B, Van der Bruggen B, Pinoy L. Influence of the type of anion membrane on solvent flux and back diffusion in electrodialysis of concentrated NaCl solutions, Chem. Eng. Sci. 113 (2014) 95100.

15 Nakanishi K, Solomon PH. Infrared Absorption Spectroscopy, 2d ed, San Francisco : Holden-Day, 1977.

16 Kuć M, Cies̈lik-Boczula K, Wwiątek P, Jaszczyszyn A, Gąsiorowski K, Malinka W. FTIR-ATR study of the influence of the pyrimidine analog of fluphenazine on the chain-melting phase transition of sphingomyelin membranes, Chem. Phys. 2015;458:9-17.

17 Grochowicz M, Kierys A. TG/DSC/FTIR studies on the oxidative decomposition of polymer-silica composites loaded with sodium ibuprofen, Polym. Degrad. Stab. 2017;138:151-160.

18 Amado FDR, Rodrigues MAS, Morisso FDP, Bernardes AM, Ferreira JZ, Ferreira CA. High-impact polystyrene/polyaniline membranes for acid solution treatment by electrodialysis: Preparation, evaluation, and chemical calculation, J. Colloid Interface Sci. 2008;320:52-61.

19 Cheng J, Yang X, Dong L, Yuan Z, Wang W, Wu S, Chen S, Zheng G, Zhang W, Zhang D, Wang $\mathrm{H}$. Effective nondestructive evaluations on UHMWPE/RecycledPA6 blends using FTIR imaging and dynamic mechanical analysis, Polym. Test. 2017;59:371-376.

20 Garcia-Vasquez W, Ghalloussi R, Dammak L, Larchet C, Nikonenko V, Grande D Structure and properties of heterogeneous and homogeneous ion-exchange membranes subjected to ageing in sodium hypochlorite, J. Memb. Sci. 2014;452:104-116.

21 Guan R, Zou H, Lu D, Gong C, Liu Y. Polyethersulfone sulfonated by chlorosulfonic acid and its membrane characteristics, Eur. Polym. J. 2005;41:1554-1560.

22 Wu KH, Wang YR, Hwu WH. FTIR and TGA studies of poly ( 4-vinylpyridine- co divinylbenzene )- Cu ( II ) complex, Polym. Degrad. Stab. 2003;79:195-200. 
23 Ongkowijaya N, Sect C, Dunn TAN. Polymer IR Spectroscopy Nicholas Ongkowijaya, 2012:1-19.

24 Kabbany FEI, Taha S, Hafez M. IR spectroscopic analysis of polymorphism in C13H14N4O., Spectrochim. Acta. A. Mol. Biomol. Spectrosc. 2011;78:981-988.

25 Krishnakumar V, Balachandran V. FTIR, FT-Raman spectral analysis and normal coordinate calculations of 2-hydroxy-3-methoxybenzaldehyde thiosemicarbozone, Indian J. Pure Appl. Phys. 2004;42:313-318.

26 Hébert $P$, Rille AL, Zheng WQ, Tadjeddine A. Vibrational spectroscopic study of the adsorption of pyridine at the $\mathrm{Au}(111)$-electrolyte interface by in situ difference frequency generation, J. Electroanal. Chem. 1998;447:5-9. 